\title{
Photoresponsive Hydrogels as Drug Delivery Systems
}

\author{
Celine DG. Abueva ${ }^{1}$ \\ Phil-Sang Chung ${ }^{1,2}$ \\ Hyun-Seok Ryu ${ }^{1}$ \\ So-Young Park ${ }^{1}$ \\ Seung Hoon Woo ${ }^{1,2}$ \\ 'Beckman Laser Institute Korea, Dankook
University College of Medicine, Cheonan, Korea
${ }^{2}$ Department of Otorhinolaryngology-Head and
Neck Surgery, Dankook University College of
Medicine, Cheonan, Korea
}

Received May 30, 2020

Accepted June 4, 2020

\section{Correspondence}

Seung Hoon Woo

Department of Otorhinolaryngology - Head and Neck Surgery, Dankook University College of Medicine, 201 Manghyang-ro, Dongnam-gu,

Cheonan 31116, Korea

Tel.: +82-41-550-178

Fax: +82-41-550-7837

E-mail: lesabyahanmail.net

(C) Korean Society for Laser Medicine and Surgery

(c) This is an open access article distributed under the terms of the Creative Commons Attribution NonCommercial License (http://creativecommons.org/ licenses/by-nc/4.0) which permits unrestricted noncommercial use, distribution, and reproduction in any medium, provided the original work is properly cited.
Hydrogels have been developed and used in tissue engineering and regenerative medicine to deliver therapeutics to injured or diseased tissue because of their versatility and properties that can be tailored to match the natural extracellular matrix. Hydrogels can be made with a variety of physical and chemical properties combined with light responsiveness ideal for applications in different fields of medicine that require the spatiotemporal control of therapeutics. Light, as a stimulus, is relatively inexpensive, contact-free, noninvasive with high spatial resolution and temporal control, convenient and easy to use, and allows deep tissue penetration that is relatively harmless. Photoresponsive hydrogels are ideal candidates for on-demand drug delivery systems that are capable of sustained and controlled drug release, minimizing the side effects, and ensuring the activity and efficient delivery of drugs to the target tissue.

\section{Key words}

Light; Laser; Photoresponsive; Hydrogel; Drug delivery 


\section{INTRODUCTION}

Hydrogels are cross-linked polymeric networks based on hydrophilic macromonomers that are able to retain large amounts of water. ${ }^{1}$ They are excellent soft materials with tunable chemical structure and physical properties as well as good biocompatibility, biodegradability, limited or minimal toxicity, and simplified synthesis methods. ${ }^{2,3}$ Over the years, hydrogels have evolved from the classical ones to intelligent ones, capable of responding to external stimuli such as $\mathrm{pH}$, light, electricity, magnetic field and enzymes with the proper selection of polymer composition and assembly. This has led to its diverse applications in various fields. In particular, hydrogels have been of great interest in drug delivery, which have been developed in many branches of medicine, including cardiology, oncology, immunology, wound healing, and pain management. ${ }^{4-8}$

Drug release in response to stimuli or physiologic changes would be an ideal delivery system, wherein drug release is highly controlled and non-specific side effects at the off-target sites are alleviated. These were some critical issues with conventional drug delivery systems that had systemic toxicity and repeated dosing. In contrast to stimuli responsive hydrogels that would serve as drug delivery vehicles with no systemic toxicity and controlled dosing. Stimuli responsive hydrogels ensure that the therapeutic benefits from the drug are optimized. With their tunable physical properties that confer controlled drug release features and ability to protect the drug from degradation, stimuli responsive hydrogels emerge as highly efficient drug delivery systems.

A variety of stimuli responsive hydrogels with delicately controlled physical and chemical properties have been developed in the past decades. ${ }^{9,10}$ Among these are the photoresponsive hydrogels that have gained significant attention largely owing to their amenability to light-induced modulation and their minimal invasiveness for in vivo and clinical applications. Herein we discuss photoresponsive hydrogels and how they could be manipulated and applied for targeted drug delivery applications. Suitable examples from the literature are provided that support the recent advancements of photoresponsive hydrogels in targeted drug delivery in diverse disease areas, and how they could be suitably made to achieve significant impact in targeted drug delivery. Photoresponsive hydrogels with their versatility are promising delivery vehicles of therapeutic molecules in several disease conditions. ${ }^{11,12}$

\section{LIGHT AS STIMULUS}

Interaction of light with photoresponsive hydrogels

Light as stimulus for drug delivery systems is advantageous due to its noninvasive nature, high spatial resolution and temporal control, and convenience and ease of use. For these reasons, light has been extensively applied in a variety of biomedical applications beyond drug delivery, including image-guided surgery, polymerization and degradation of tissue engineering scaffolds, and photodynamic therapy for cancer. ${ }^{13,14}$ In this review, the scope has been limited to light-triggered drug delivery systems that have demonstrated triggered cargo release for each of the major light-responsive drug delivery mechanisms: photochemically triggered release, photoisomerization and photothermal release (Fig. 1). The many mechanisms utilized for light-triggered responses has been previously reviewed. ${ }^{15-17}$ Often, photoresponsive hydrogels incorporate photocleavable or photoisomerizable components that allow light-mediated changes in their properties (Table 1). The interaction of light with photoresponsive hydrogels can result in different responses. Depending on the location and type of photoresponsive moiety, lightinduced responses could lead to swelling or shrinking, crosslinking or de-crosslinking, degradation or permanent chemical modification, etc. Examples are highlighted herein to illustrate progress for each mechanism, and key limitations are identified to motivate future research and advance the field.

Several works in the field involved the fabrication of photodegradable hydrogels out of different synthetic polymers by incorporating photolabile groups to the polymer backbone. The controlled photodegradation could control the release of therapeutic factor at the target area. In particular, the o-nitrobenzyl group (o-NB) is frequently utilized and incorporated in polymers. Several works have been done creating a library of polymerizable 0 nitrobenzyl macromers with varying functionalities to allow for direct conjugation to various bioactive molecules and polymers. ${ }^{18}$ Crosslinks containing o-nitrobenzyl groups were also used to hold together hydrogels made of polyethylene glycol (PEG) and polyacrylamide (PAM) that when undergoes photodegradation results in release of the model protein. One study incorporated up to three different photocleavable groups into the backbone of PEG macromers to make photodegradable hydrogels. ${ }^{18}$ All of the photocleavable groups contained the o-NB moiety but they each had varying modifications that changed their reactivity to different wavelengths of light. When exposed to low intensity $\left(<45 \mathrm{~mW} / \mathrm{cm}^{2}\right)$ wavelengths of light rang- 


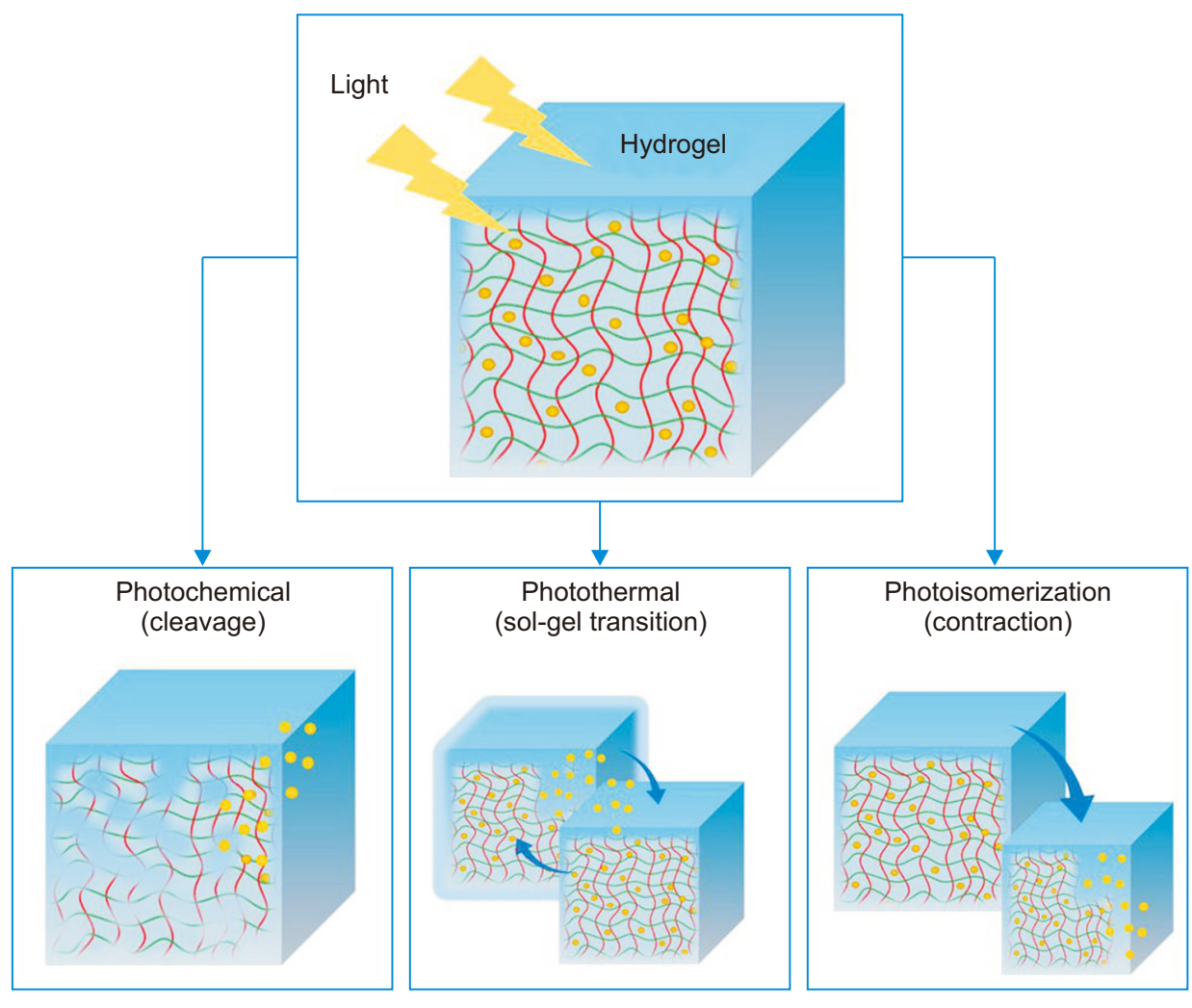

Fig. 1. Mechanisms involved in the light-triggered release of therapeutics in a photoresponsive hydrogel.

Table 1. Lists of some functional groups used in the design of photoresponsive hydrogels that undergo cleavage and isomerization in response to light

\begin{tabular}{|c|c|c|c|}
\hline Photoreaction \& Functional Group & Chemistry (e.g.) & Wavelength & Ref. \\
\hline \multicolumn{4}{|l|}{ Cleavage } \\
\hline Nitrobenzyl estser & & $365 \mathrm{~nm}$ & 18,32 \\
\hline Coumarin & & $>365 \mathrm{~nm}$ & 33,34 \\
\hline \multicolumn{4}{|l|}{ Isomerization } \\
\hline \multirow[t]{3}{*}{ Azobenzene } & & $365 \mathrm{~nm}, 445 \mathrm{~nm}$ & 35,36 \\
\hline & & $265 \mathrm{~mm} \mathrm{Vir}$ & \\
\hline & & $365 \mathrm{~nm}$, VIS & 36 \\
\hline
\end{tabular}

Vis, visible light. 
ing from 365 to $436 \mathrm{~nm}$ for $5 \mathrm{~min}$, the authors demonstrated the triggered sequential release of three different dyes (fluorescein, rhodamine and aminomethylcoumarin acetate). In another study, Liu and their co-workers ${ }^{19}$ designed and synthesized a photo and $\mathrm{pH}$ dual-sensitive amphiphilic copolymers with photolabile o-NB groups that can self-assemble into stimuli-regulated amphiphilic micelles in aqueous solution carrying doxorubicin (DOX) for cancer therapy. The DOX-loaded micelles showed a cumulative release ratio of $10.09 \mathrm{wt} \%$ at $\mathrm{pH} 5.0$ after UVirradiation for $20 \mathrm{~min}$. A high cumulative DOX release ratio $(74.70 \%)$ at the simulated tumor microenvironment was achieved within 6 days. ${ }^{20}$ Aside from photodegradable hydrogels, hydrogels with photocontrollable cell adhesion molecules to control biomolecule density and degree of cell attachment to the surface have been prepared utilizing similar photocleavable nitrobenzyl moiety. ${ }^{21}$ Photoresponsive hydrogels have also been investigated and found to be capable of encapsulating and releasing live cells ondemand. ${ }^{22}$

These studies have shown the advantages of using light as a stimulus to control the release of therapeutic agents: drugs, proteins and other biomolecules, control cell attachment and growth with adaptive surfaces, and even carry live cells.

\section{Controlled and targeted drug release}

Controlled drug release is essential for improving therapeutic efficacy and minimizing adverse effects due to the strong targeted features to regulate the distribution of drugs in vivo. Photoresponsive hydrogels can provide spatial and temporal control in the release of drugs or therapeutics. The 3D networks of hydrogels allow loading of drugs, while its light-induced gel-to-sol transition can be used to release the loaded drug. ${ }^{23}$

Recently, NIR-responsive hydrogels (Table 2) have been of interest as they provide great potentials for therapeutic treatment due to both the low health hazard and deeper light penetration in tissue. In an example, Cao and co-workers developed an NIR-light-controlled drug delivery platform based on the composite hydrogels of low-melting-point agarose and PEGylated black phosphorous (BP) nanosheets. ${ }^{24}$ In this study, NIR-induced $(808 \mathrm{~nm})$ heating due to the photothermal conversion of $\mathrm{BP}$ resulting in hydrolysis and subsequent melting of the hydrogel that triggers the controlled release of encapsulated doxorubicin (DOX), a cancer treatment drug. In vivo experiments with tumor tissues also demonstrated this system to be capable of controlling the release of drugs to reduce and eliminate subcutaneous breast and melanoma cancers. The hydrogel is biodegradable and easily excreted through urine after the treatment, which is promising for clinical translation. Further advancement in NIR-responsive hydrogels has led to the development of upconversion nanoparticles, wherein luminescence converts NIR light into UV light. Zhao and co-workers introduced upconversion nanoparticles into PEG hydrogels with photolabile linkers. ${ }^{25} \mathrm{NIR}$ light $(980 \mathrm{~nm})$ could then induce the gel-sol transition and trigger the release of biomacromolecules (proteins and enzymes) entrapped in the hydrogel without sacrificing their biocompatibility. These upconversion nanoparticles have been studied and are continually being developed for photodynamic therapy in cancer due to their ability to deliver chemotherapeutic drugs in to the tumor microenvironment. ${ }^{26,27}$ The use of light or laser to control the release and activation of chemotherapeutic drugs on site reducing the toxicity towards healthy surrounding cells or tissue have made such an impact on cancer research.

\section{Laser assisted drug delivery}

Lasers have been investigated and used clinically to

Table 2. Other examples of near-infrared (NIR)-responsive hydrogels/ structures that have been developed as delivery systems and the significant findings

\begin{tabular}{|c|c|c|}
\hline Type & Detail & Ref \\
\hline Upconversion nanoparticles & $\begin{array}{l}\text { Continuous-wave NIR light ( } 980 \mathrm{~nm} \text { ) can be used to induce the sol-gel transition and release large, } \\
\text { inactive biomacromolecules (protein and enzyme) entrapped in the hydrogel into aqueous solution } \\
\text { "on demand" }\end{array}$ & 25 \\
\hline Injectable supramolecular hydrogel & $\begin{array}{l}\text { NIR light-responsive supramolecular hydrogel consisting of } \alpha \text {-cyclodextrin and polyethylene glycol } \\
\text { CPEG-modified dendrimer-encapsulated platinum nanoparticles release the entrapped therapeutic } \\
\text { agents in an on-demand and dose-tunable fashion }\end{array}$ & 37 \\
\hline Composite hydrogel & $\begin{array}{l}\text { Dopamine nanoparticle (DP) was incorporated as a highly effective photothermal agent as well as } \\
\text { anticancer drug, bortezomib (BTZ) carrier inside a stimuli-responsive pNIPAAm-co-pAAm hydrogel }\end{array}$ & 38 \\
\hline $\begin{array}{l}\text { PEG-NH2, ssDNA-caged } \\
\text { aptamer nanostructure }\end{array}$ & NIR light-activated cancer cell targeting and doxorubicin delivery in photothermal therapy & 39 \\
\hline
\end{tabular}


enhance the delivery of therapeutic agents that requires deeper penetration. For instance, topical drug delivery, which is essential to dermatological therapy. The cutaneous bioavailability of most topically applied drugs is relatively low with only $1-5 \%$ being absorbed into the skin, and some drugs that do get absorbed do not penetrate deeply enough to reach the desired target in the tissue. ${ }^{28}$ Many medications are too large to penetrate the stratum corneum barrier and require either an injectable or systemic delivery. Laser assisted drug delivery is an evolving modality that could allow for a greater depth of penetration by existing topical medications, and a more efficient method for transcutaneous delivery of large drug molecules. ${ }^{29}$ One study is the use of laser-assisted delivery to enhance less-permeable drugs and cosmeceuticals in targeting cutaneous lesions for both efficacy and safety. ${ }^{30}$ Another study investigated the effect of combining laser and application of topical whitening agent together to treat Melasma, a common hyperpigmentation disorder. ${ }^{31}$ These studies have found significantly enhanced penetrations to a certain extent. But the majority of the existing studies on laser assisted drug delivery have been performed on animal models and additional human studies are needed.

\section{CONCLUSIONS AND FUTURE PERSPECTIVES}

For the past decades, hydrogels have evolved from the simple chemically or physically cross-linked networks loaded with a single component to the present with complex multicomponent systems capable of releasing multiple therapeutics in a spatially and temporally controlled and triggered manner. The diversity of hydrogel chemical composition and structure combined with a responsiveness to light, a stimulus with various advantages such as being cheap, contact free, and spatiotemporally controllable make photoresponsive hydrogels ideal candidates for applications in a wide range of fields. Although substantial effort has been dedicated to the design and investigation of photoresponsive hydrogels in different fields, most of the present systems are restricted to proof-of-concept studies. Many have demonstrated successes as in vitro, and some have produced promising preclinical in vivo results. However, there have not been any clinical trials for photoresponsive drug delivery systems. This is partly due to the complex design of many systems. Thus, going forward, it will be advantageous to keep the system design simple and straightforward for clinical translation. Nonetheless, we believe that photoresponsive hydrogels will increase their role within the field of therapeutic delivery with the many exciting approaches currently under way to advance the clinical effectiveness of photoresponsive drug delivery systems.

\section{ACKNOWLEDGEMENTS}

We thank Beckman Laser Institute Korea in Dankook University for the support in completing this manuscript.

\section{CONFLICT OF INTEREST}

The authors have no conflict of interest to disclose.

\section{REFERENCES}

1. Buwalda SJ, Vermonden T, Hennink WE. Hydrogels for therapeutic delivery: current developments and future directions. Biomacromolecules 2017;18:316-30.

2. Drury JL, Mooney DJ. Hydrogels for tissue engineering: scaffold design variables and applications. Biomaterials 2003;24:433751.

3. Wang K, Hao Y, Wang Y, Chen J, Mao L, Deng Y, et al. Functional hydrogels and their application in drug delivery, biosensors, and tissue engineering. Int J Polym Sci 2019;2019:3160732.

4. Chao Y, Chen $Q$, Liu Z. Smart injectable hydrogels for cancer immunotherapy. Adv Funct Mater 2020;30:1902785.

5. Fan DY, Tian Y, Liu ZJ. Injectable hydrogels for localized cancer therapy. Front Chem 2019;7:675.

6. Liu H, Wang C, Li C, Qin Y, Wang Z, Yang F, et al. A functional chitosan-based hydrogel as a wound dressing and drug delivery system in the treatment of wound healing. RSC Adv 2018;8:7533-49.

7. Mateus D, Marto J, Trindade P, Gonçalves H, Salgado A, Machado $P$, et al. Improved morphine-loaded hydrogels for woundrelated pain relief. Pharmaceutics 2019;11:76.

8. Peña B, Laughter M, Jett S, Rowland TJ, Taylor MRG, Mestroni $\mathrm{L}$, et al. Injectable hydrogels for cardiac tissue engineering. Macromol Biosci 2018;18:e1800079.

9. Cai Z, Gan Y, Bao C, Wu W, Wang X, Zhang Z, et al. Photosensitive hydrogel creates favorable biologic niches to promote spinal cord injury repair. Adv Healthc Mater 2019;8:e1900013.

10. Tomatsu I, Peng K, Kros A. Photoresponsive hydrogels for biomedical applications. Adv Drug Deliv Rev 2011;63:1257-66.

11. Caló E, Khutoryanskiy W. Biomedical applications of hydrogels: a review of patents and commercial products. Eur Polym J 2015;65:252-67.

12. Narayanaswamy R, Torchilin VP. Hydrogels and their applications in targeted drug delivery. Molecules 2019;24:603.

13. Deng K, Li C, Huang S, Xing B, Jin D, Zeng Q, et al. Recent progress in near infrared light triggered photodynamic therapy. 
Small 2017; 13:1702299.

14. Dou Q, Kenny Low ZW, Zhang K, Loh XJ. A new light triggered approach to develop a micro porous tough hydrogel. RSC Adv 2017;7:27449-53.

15. Alvarez-Lorenzo C, Bromberg L, Concheiro A. Light-sensitive intelligent drug delivery systems. Photochem Photobiol 2009;85:848-60.

16. Fomina N, Sankaranarayanan J, Almutairi A. Photochemical mechanisms of light-triggered release from nanocarriers. Adv Drug Deliv Rev 2012;64:1005-20.

17. Mura S, Nicolas J, Couvreur P. Stimuli-responsive nanocarriers for drug delivery. Nat Mater 2013;12:991-1003.

18. Zhao H, Sterner ES, Coughlin EB, Theato P. o-Nitrobenzyl alcohol derivatives: opportunities in polymer and materials science. Macromolecules 2012;45:1723-36.

19. Zhao X, Qi M, Liang S, Tian K, Zhou T, Jia X, et al. Sensitive amphiphilic copolymer PEG43-b-P(AA76-co-NBA35-co-tBA9) and its micellization as leakage-free drug delivery system for UVtriggered intracellular delivery of doxorubicin. ACS Appl Mater Interfaces 2016;8:22127-34.

20. Xin F, Wei M, Jiang S, Gao Y, Nie J, Wu Y, et al. Design of hydrophilic photocleavage o-nitrobenzyl acrylate-modified nanogels with outstanding biocompatibility prepared by RAFT polymerization for drug carrier. Eur Polym J 2020;122:109364.

21. Wegner SV, Sentürk OI, Spatz JP. Photocleavable linker for the patterning of bioactive molecules. Sci Rep 2015;5:18309.

22. Griffin DR, Kasko AM. Photodegradable macromers and hydrogels for live cell encapsulation and release. J Am Chem Soc 2012;134:13103-7.

23. Li L, Scheiger JM, Levkin PA. Design and applications of photoresponsive hydrogels. Adv Mater 2019;31:e1807333.

24. Qiu M, Wang D, Liang W, Liu L, Zhang Y, Chen X, et al. Novel concept of the smart NIR-light-controlled drug release of black phosphorus nanostructure for cancer therapy. Proc Natl Acad Sci U S A 2018;115:501-6.

25. Yan B, Boyer JC, Habault D, Branda NR, Zhao Y. Near infrared light triggered release of biomacromolecules from hydrogels loaded with upconversion nanoparticles. J Am Chem Soc 2012;134:16558-61.

26. Hamblin MR. Upconversion in photodynamic therapy: plumbing the depths. Dalton Trans 2018;47:8571-80.

27. Lee SY, Lee R, Kim E, Lee S, Park YI. Near-infrared lighttriggered photodynamic therapy and apoptosis using upconversion nanoparticles with dual photosensitizers. Front Bioeng Biotechnol 2020;8:275.

28. Nino M, Calabrò G, Santoianni P. Topical delivery of active prin- ciples: the field of dermatological research. Dermatol Online J 2010;16:4.

29. Sklar LR, Burnett CT, Waibel JS, Moy RL, Ozog DM. Laser assisted drug delivery: a review of an evolving technology. Lasers Surg Med 2014;46:249-62.

30. Leo Goo B. Laser assisted drug and cosmeceutical delivery system of the skin. Med Lasers 2015;4:51-9.

31. Kim JE, Kim JK, Ko JY, Ro YS, Chang SE. Topical application of whitening agents after erbium-doped yttrium aluminum garnet fractional laser treatment for melasma in Asians: a randomized controlled split-face study. Med Lasers 2012;1:3-10.

32. Hansen MJ, Velema WA, Lerch MM, Szymanski W, Feringa $B L$. Wavelength-selective cleavage of photoprotecting groups: strategies and applications in dynamic systems. Chem Soc Rev 2015;44:3358-77.

33. Bao C, Fan G, Lin Q, Li B, Cheng S, Huang Q, et al. Styryl conjugated coumarin caged alcohol: efficient photorelease by either one-photon long wavelength or two-photon NIR excitation. Org Lett 2012;14:572-5.

34. Ji W, Qin M, Feng C. Photoresponsive coumarin-based supramolecular hydrogel for controllable dye release. Macromol Chem Phys 2018;219:1700398.

35. Tamesue S, Takashima Y, Yamaguchi H, Shinkai S, Harada A. Photoswitchable supramolecular hydrogels formed by cyclodextrins and azobenzene polymers. Angew Chem Int Ed Engl 2010;49:7461-4.

36. Aburaya Y, Nomura H, Kageshima M, Naitoh Y, Li YJ Sugawara $Y$. Switching surface polarization of atomic force microscopy probe utilizing photoisomerization of photochromic molecules. J Appl Phys 2011;109:064308.

37. Wang $X$, Wang $C$, Zhang Q, Cheng Y. Near infrared lightresponsive and injectable supramolecular hydrogels for ondemand drug delivery. Chem Commun (Camb) 2016;52:978-81.

38. GhavamiNejad A, SamariKhalaj M, Aguilar LE, Park CH, Kim CS. pH/NIR light-controlled multidrug release via a musselinspired nanocomposite hydrogel for chemo-photothermal cancer therapy. Sci Rep 2016;6:33594.

39. Yang Y, Liu J, Sun X, Feng L, Zhu W, Liu Z, et al. Near-infrared light-activated cancer cell targeting and drug delivery with aptamer-modified nanostructures. Nano Res 2016;9:139-48.

How to cite this article: Abueva CDG, Chung PS, Ryu HS, Park SY, Woo SH. Photoresponsive hydrogels as drug delivery systems. Med Lasers 2020;9:6-11. https://doi.org/10.25289/ ML.2020.9.1.6 